\title{
Producción imaginativo-reflexiva infantil en talleres clínico-narrativos
}

\author{
Alicia Kachinovsky* \\ Hernán Correa \\ Mauro Magnone \\ Universidad de la República, Centro de Investigación Clínica en Psicología. Montevideo, Uruguay
}

\begin{abstract}
Resumen: El trabajo se centra en la variable subjetiva del aprendizaje: busca identificar las transformaciones psíquicas de seis niños en su tránsito por un dispositivo clínico mediado por cuentos (taller narrativo). El trabajo de campo se desarrolló en una escuela pública, con niños de 6 y 7 años, con pronóstico pedagógico desfavorable. Una herramienta informática organiza el análisis cualitativo y cuantitativo de la producción discursiva infantil. Los resultados ponen de manifiesto un incremento de dicha producción a medida que se fueron desarrollando los talleres y un menor requerimiento de participación del equipo coordinador. Sin embargo, se observa una tendencia decreciente de la producción imaginativo-reflexiva que motiva a focalizar el presente texto en esa dimensión y a dar cuenta de sus posibles causas.
\end{abstract}

Palabras-clave: clínica psicoanalítica, niños, taller narrativo, procesos imaginativo-reflexivos.

\section{Introducción}

Se presentan en este artículo avances de un proyecto que acoge como hipótesis de trabajo que el cuento infantil oficia como mediador simbólico para el psiquismo cuando se lo inserta en dispositivos clínicos de orientación psicoanalítica. Asume como directos antecedentes las investigaciones de tres espacios académicos liderados por Silvia Schlemenson en Argentina (Álvarez, 2010; Cantú, 2011; Schlemenson, 2009), Celso Gutfreind en Brasil (2003, 2007) y Alicia Kachinovsky en Uruguay (2014, 2016). Dicha hipótesis ha sido explorada en diversos talleres clínico-narrativos, realizados en escuelas públicas de contextos vulnerables. Su coordinación ha estado a cargo de investigadores abocados a esta temática, que practican la concepción de un psicoanálisis extramuros. El cuerpo teórico del psicoanálisis junto al análisis del discurso constituye la perspectiva teórico-metodológica adoptada.

En esta oportunidad los resultados obtenidos remiten a la edición de un taller integrado por seis niños de 6 y 7 años, con pronóstico pedagógico desfavorable, organizado a fin de construir un estudio de casos centrado en la dimensión subjetiva del aprendizaje. Todos los niños que protagonizaron el taller han vivido procesos de desintegración familiar; algunos también han experimentado situaciones de desarraigo familiar y territorial o violencia doméstica, produciéndose quiebres de su continuidad existencial. Al estar comprometidos los procesos de simbolización de estos escolares, el objetivo ha sido identificar y describir las transformaciones psíquicas ocurridas en cada uno de ellos (unidades de análisis)

*Endereço para correspondência: alika@psico.edu.uy a partir de su inclusión en el taller y a través de la producción verbal registrada.

Con este propósito se crea un esquema de anotación mediante el cual se analizan cualitativa y cuantitativamente dichas producciones, basado en una herramienta informática, MMAX2 (Müller \& Strube, 2006), para determinar la relación entre la producción discursiva infantil y las variables de orden psíquico. Se implanta así una modalidad de triangulación diferente y novedosa respecto a hallazgos anteriores. Si bien son tres las dimensiones de análisis que se han usado hasta el momento, este informe se circunscribe a los avances de una de ellas, los procesos imaginativo-reflexivos. La importancia de la dimensión en cuestión determina un tratamiento preferencial, en la medida que involucra los más caros procesos del pensamiento. Además, esta categoría comprende parcialmente a las otras dos ("construcción de alteridad", "tratamiento del conflicto").

Los resultados obtenidos ponen de manifiesto un incremento de la producción discursiva infantil a medida que se desarrollaron los talleres y un menor requerimiento de participación de los referentes adultos para alcanzar estos niveles. Simultáneamente se observa una tendencia decreciente de la producción imaginativo-reflexiva, justificada por la incidencia de la misma intervención, otro motivo para detenerse en ella.

\section{Problema de investigación}

El problema que enmarca el proyecto es el fracaso escolar y sus alternativas, fenómeno que acompaña a los sistemas escolares desde su universalización, planteando 
grandes retos al sistema educativo y al conjunto de la sociedad. El fracaso escolar suele ser una forma de exclusión social temprana que se debe leer en clave de responsabilidad institucional, descentrando al aprendiz del lugar de responsable (Kachinovsky, 2012). A pesar de su carácter complejo y multidimensional (político, social, institucional, pedagógico, psicológico, etc.), el peso relativo de estos factores varía caso a caso. Un abordaje personalizado, como el que se presenta, procura ser respetuoso de esta diversidad. Por eso se circunscribe a una de las variables identificadas, los procesos de simbolización y sus fracturas. Este recorte prioriza el malestar padecido por algunos niños con motivo de experiencias dramáticas no metabolizadas (no entramadas a la malla representacional), no semantizadas. El malestar se expresa en cada niño de diferentes modos (inhibiciones, marcada inquietud, sentimiento de sí devaluado, restricciones imaginativas, exigua autonomía, verborragia, pensamiento fabulador, rigidez intelectual, conflictos con pares, etc.), características que menoscaban el desempeño escolar, agravando el sufrimiento. Se trata de reemplazar una posición anclada en el niño de la carencia -que hace del fracaso escolar una profecía autocumplidapor el sujeto de la educación o sujeto de la posibilidad (Martinis, 2013; Patto, 1988).

Se entiende por procesos de simbolización aquellas operaciones mentales responsables de la actividad representativa, es decir, la apropiación simbólica del objeto ausente. Esto requiere mecanismos sustitutivos, privativos de una relación de equivalencia: el símbolo representa a la cosa, pero no es la cosa en sí.

A través del taller clínico-narrativo se pretende instituir o restituir una disposición favorable hacia la cultura letrada. El camino que conduce a su acceso se sostiene, a su vez, en requisitos psíquicos insoslayables. De esta forma se aspira a contribuir a la prevención y abordaje del fracaso escolar, colaborando a la diversificación de alternativas requerida por este problema (Administración Nacional de Educación Pública [ANEP], 2009).

El objetivo de las intervenciones clínicas en el campo educativo consiste en propender a la complejización de los procesos simbólicos y sus respectivas producciones, es decir, los modos en que un niño o un adolescente construye sus conocimientos, comprende lo que lee, escribe o se expresa gráficamente. Sus estrategias apuntan al despliegue de la imaginación y del pensamiento autónomo en el campo social. Los niños y adolescentes que consultan por sus dificultades de aprendizaje suelen presentar un menoscabo de su imaginación, de su interrogación crítica y de la autonomía de su pensamiento. En el mejor de los casos, sus narraciones son descriptivas (reproduccionistas) e incompletas.

El trabajo pedagógico con cuentos procura la recuperación de la información transmitida (personajes, contextos, argumentos), la comprensión y discernimiento de eventuales valores y el reconocimiento del repertorio lexical disponible. En la perspectiva del taller clínico en el que se utiliza el cuento como mediador, sus cometidos son otros. Se trabaja con grupos de 4 a 6 niños, cuyas restricciones simbólicas muestran un fuerte anclaje emocional a priorizar. Las piezas literarias compartidas con el pequeño auditorio constituyen un peculiar punto de partida, ya que suelen modelar los conflictos humanos universales que los inspiran y las aflicciones consecuentes, a la vez que preservan la intimidad o fragilidad del lector/oyente. Mantienen asimismo suficiente distancia con el mundo real, de modo de no agobiar a quien padece afecciones similares. Siempre desde el enfoque definido, estas piezas cumplen un papel de ligazón intrapsíquica e intersubjetiva, sustrato de los procesos de simbolización. La lectura de esas historias persigue el fin de anudar o articular lo relatado con lo propio de cada uno, de enlazar las representaciones ficcionales con las experienciales y engrosar así la malla representativa por donde se desplazan y tramitan los afectos.

Los cambios observados en la producción discursiva de los casos estudiados reflejan las transformaciones de los procesos psíquicos subyacentes. En un entorno de mayor bienestar, se apuesta a cultivar la apetencia narrativa y a atenuar aquellos factores que obstaculizan el deseo de saber. Los efectos sobre el campo pedagógico son, por lo tanto, indirectos.

Con la idea de seguir poniendo a prueba algunas hipótesis y resultados previos, el objetivo trazado fue crear una herramienta de análisis cualitativo y cuantitativo que indagara y especificara la relación entre la producción discursiva infantil y las dimensiones de orden psíquico. Para satisfacer dicho propósito, era necesario diseñar un marco de anotación aplicable a un corpus de discurso infantil que relacionara categorías psíquicas con categorías discursivas y crear un corpus de discurso infantil a partir de lo registrado en los talleres clínico-narrativos.

\section{Marco teórico}

La presente investigación, desarrollada en un ámbito escolar, descentra la cuestión educativa de lo meramente cognitivo y sitúa sus preguntas alrededor de la relación con el saber, cuando esta relación es mediatizada por el cuento infantil. Éste es un reservorio de contribuciones simbólicas de diferente índole:

- un surtido lingüístico compuesto por aportes semánticos y morfosintácticos;

- una variedad de conflictos humanos de los que se nutre y a la vez modela, ofreciendo perspectivas y alternativas diferentes;

- una miscelánea de estados afectivos que presenta o sugiere, convocando la sensibilidad y singularidad del oyente/lector;

- una experiencia estética modalizada por el «como si» de la ficción, que allana el camino para afrontar situaciones difíciles de metabolizar. 
La concomitancia cognitivo-afectiva que caracteriza estas obras de la literatura es condición de viabilidad de los procesos de simbolización, haciendo de la narrativa una herramienta idónea para el trabajo clínico con niños que manifiestan restricciones en su actividad representativa, expresadas en términos de dificultades escolares. El taller clínico-narrativo funciona entonces como estrategia aventajada para recomponer la relación con el saber sobre el sí mismo y sobre el mundo. Cada historia relatada es concebida como un potencial objeto de conocimiento, siendo requisitos de su aprehensión la construcción subjetiva de su alteridad y un $Y o$ suficientemente cohesionado que lo invista y se aboque a su apropiación mental. Cuando un sujeto puede hacer uso del objeto (Winnicott, 1989/1991), dicho objeto se habrá de configurar por medio de tres operaciones posibles, a saber: uso reproductivo, uso interpretativo, uso creativo (Kachinovsky, 2016). Esta última modalidad es la que da lugar a los procesos imaginativo-reflexivos.

Más allá de importantes disidencias dentro del campo filosófico, se considera en este trabajo que los conceptos de imagen e imaginación están íntimamente vinculados entre sí, ya sea en sus versiones conscientes como en las inconscientes. Desde este punto de vista, la imaginación opera tanto en el campo de la percepción como en el de la creación (Warnock, 1978). Esto se relaciona con la hipótesis según la cual la imaginación participa en todos los niveles de la actividad representativa. Por un lado, amarra al sujeto al mundo, situándolo y contactándolo con lo compartible de la otredad. Por otro lado, siempre ligada a sentimientos y emociones, permite ir más allá de lo percibido, estableciendo inferencias e inventando (Xirau, 2018).

El campo social, la escuela o el taller en este caso ofrece objetos susceptibles de inversión. El texto narrativo es uno de ellos. Al ser compartido, se presenta como un activador de imágenes (si ha logrado atraer la atención del destinatario). Cada imagen, lejos de limitarse a copiar la realidad leída o escuchada, entraña sentidos novedosos, "que articulan restos perceptivos, trazos semióticos de afectos, con dimensiones intersubjetivas y verbales" (Wald, 2015). La incitación a producir asociaciones, posterior a la lectura de cuentos, provoca una revuelta anímica de intensidad variable. La movilización conseguida es, con frecuencia, una oportunidad de cambio.

La imaginación es entonces una facultad humana que se manifiesta a diferentes niveles y modalidades, desde el reconocimiento de objetos (imaginación primaria) hasta la creación de obras artísticas (imaginación secundaria), en presencia y en ausencia del objeto respectivamente (Warnock, 1994). Estas categorías responden a un particular gradiente de creación o poiesis.

Bajo un enfoque psicoanalítico, dicho gradiente es advertido y justificado a través de un modelo de aparato psíquico en el que se produce una cooperación intersistémica versátil, según la tarea y el sujeto en cuestión. En cuanto a la tarea, lo imaginativo se manifiesta en las creaciones ficcionales gráficas o verbales, en la producción onírica y en las diferentes manifestaciones artísticas (cine, escultura, pintura etc.). En cuanto a la singularidad del acto imaginativo, es necesario recurrir a la primera tópica freudiana, a sus principios y dinamismos. En este sentido, el comercio entre el sistema inconsciente (regido por los procesos primarios) y el preconsciente-consciente (regido por los procesos secundarios), alcanzaría su mayor expresión al dar cabida a los procesos terciarios (Green, 2003/2005). La función de estos últimos es precisamente la de articular o poner en relación a los otros dos procesos, coartando la saturación de cualquiera de ellos y protegiendo del dominio exclusivo de unos sobre otros. El pensamiento se sostiene por los procesos secundarios, responsables de las funciones cognitivas, que a su vez son gobernados por el principio de realidad. Sin embargo, toda actividad racional mantiene una apertura a los procesos primarios, garantes de una eventual intuición creadora. Al mismo tiempo, no deben contrariarse entre ellos: "Cuando pienso o escribo, necesito liberarme del asalto de los procesos primarios para asegurar un funcionamiento intelectual no trabado por la irrupción del quantum de afectos incontrolables ..." (Green, 1995/1996, p. 187). La alternancia o cuota de protagonismo de estos procesos varía de acuerdo a la persona y a la circunstancia de que se trate, mientras que la emergencia de lo novedoso depende del trabajo de ligazón oficiada por los procesos terciarios.

A nivel individual, los procesos imaginativos son menoscabados tanto por la represión como por mecanismos más radicales que la rebasan. La intensidad del afecto puede quebrantar los diques impuestos por la represión y anular la capacidad de ligazón del yo, trabando así la religazón de los afectos en producciones psíquicas de mayor complejidad (Green, 1999).

La censura social también hace lo suyo. Bajo una legítima aspiración a preservarse y perpetuarse, la sociedad fabrica sujetos que razonan y valoran según como la misma sociedad los ha configurado. Para ello se vale de una represión sutil, pero efectiva. Esta matriz social del intelecto abona un alto costo: la domesticación de la imaginación y la restricción de la capacidad de discernimiento. Cuando se afirma que el pensamiento consciente se forja en la clausura y se desenvuelve "bajo el signo de la repetición" (Castoriadis, 1991/1993, p. 43), se admite la dificultad para cuestionar un instituido que responda a un supuesto consenso social. La verdadera reflexión requiere que se impugnen las creencias socialmente sancionadas y se abandonen las certezas de la conciencia. La emergencia de un pensamiento creativo y reflexivo requiere desconfiar del saber oficial, autorizado por los otros (saber instituido), para examinar lo incuestionable. 


\section{Metodología}

\section{Caracterización}

La presente investigación adopta el enfoque clínico de investigación en ciencias humanas. Por eso privilegia el estudio de casos, entendido como método que analiza en extensión una unidad integral para responder al planteo de una interrogante o problema, ensayar hipótesis y desarrollar teoría (Hernández Sampieri, Fernández Collado, \& Baptista Lucio, 2010).

La intervención clínica estuvo a cargo de una coordinadora y dos asistentes (observadoras participantes). Se contempló un conjunto de acciones sinérgicas:

- Intercambios regulares con la maestra de clase;

- Observación de cada niño en el salón de clase;

- Observación de cada niño en el recreo;

- Estudios diagnósticos I y II (pre y post intervención);

- Entrevistas con referentes adultos (madres, padres, abuelos o equivalentes);

- Coordinación de un taller clínico-narrativo (intervención propiamente dicha).

Las observaciones de clase y recreo se cumplieron por otros asistentes, sin la figura de la coordinadora.

\section{Población objetivo}

Los talleres se llevaron a cabo con un grupo de seis niños de primer año, cuya maestra predecía un inminente fracaso escolar, en una escuela pública de tiempo completo (ETC) de la ciudad de Montevideo. Las ETC se caracterizan por presentar bajos rendimientos educativos, altas tasas de repetición en primer año, asistencia insuficiente y abandono intermitente. Su objetivo es brindar una atención de calidad a niños que viven en condiciones de pobreza, en hogares con escasos recursos culturales y situaciones de conflictividad. De acuerdo con la maestra de clase, el grupo se seleccionó según criterios de inclusión y exclusión.

Criterios de inclusión: cursar primer año por primera o segunda vez y presentar dificultades incipientes en los procesos de alfabetización vinculadas a problemas familiares o a historias de vida marcadas por experiencias traumatogénicas. Se proporcionan ejemplos para orientar a la maestra: niños con escaso surtido lexical o sintáctico (Bernstein, 1989), que no acompañan el ritmo general de su grupo (no atienden, no entienden o no pueden dar cumplimiento a las consignas), que muestran signos de inhibición o exaltación verbal, marcada inquietud o reiterados conflictos con sus pares.

Criterios de exclusión: presunción o diagnóstico de patologías severas (trastornos del desarrollo, discapacidades, trastornos graves de conducta, psicosis etc.).

\section{Sobre las intervenciones}

Los niños trabajaron semanalmente, en horario y local escolar, en un espacio de 40 a 50 minutos de duración. Se llevaron a cabo diecisiete encuentros. Debido a la edad y dificultades de los niños el énfasis se puso en la oralidad: los cuentos, poemas y canciones eran transmitidos por la coordinadora e investigadora, prestando especial atención a los matices expresivos.

La lectura del cuento era seguida por asociaciones espontáneas de los niños, que explicitaban interrogantes, cuestionamientos, afinidades o rechazos provocados por el texto. La coordinadora formulaba preguntas abiertas para (re)crear el cuento (no para reproducirlo) o proponía actividades narrativas complementarias (por ejemplo, construir otros desenlaces). Sus comentarios y señalamientos debían propiciar la apertura del campo discursivo, incluso la impugnación de los aspectos canónicos (Bruner, 1990/1991, 2002/2003) o maniqueos del cuento.

La recreación de la historia leída perseguía la obtención de versiones narrativas alternativas, a título de una reescritura de lo contado, entramadas con la identidad de los niños, con sus vicisitudes y vivencias personales y escolares. Se impulsaba asimismo la manifestación de argumentaciones e interpretaciones de lo sucedido.

\section{Acerca de los cuentos leídos}

Se contaba con un variado surtido de historias. La propia dinámica grupal determinaba cuál se elegía en cada ocasión. Con frecuencia se instauraban debates narrativos, alentados por la coordinadora, cuando surgían espontáneamente diferentes puntos de vista en el auditorio.

Se describen a continuación dos momentos del taller que ilustran algunas de las dramáticas subjetivas emplazadas. El primero de ellos remite al tercer encuentro. Los primeros momentos se dedicaron a recordar los cuentos trabajados hasta ese momento: "El joven cangrejo" y "El perro que no sabía ladrar", de Gianni Rodari. Una vez que los niños evocaron los infortunios sufridos por ambos personajes, se les preguntó qué tenían en común estas dos historias, en qué se asemejaban. La respuesta pretendida lucía simple, circunscripta a lo que ellos mismos habían mencionado y a la esfera del saber: el cangrejo no sabía caminar para adelante y el perro no sabía ladrar. Pero no resultaba tan simple para ellos, que no ocultaron su perplejidad. Superado el primer desconcierto, una niña afirmó:

Sara: Que el perro estaba solo y al cangrejo lo echaron de su casa.

Es evidente que los niños no responden según las expectativas o presunciones de los coordinadores. Por otra parte, la contestación de Sara es inobjetable: acorde con los relatos y con su sensibilidad. Su mayor preocupación era la soledad de los personajes. 
Luego, un conjunto de sentimientos piadosos tiñeron el escenario discursivo del taller:

Coordinadora: ¡Ah, qué interesante! Yo no habia pensado eso, pero es cierto.

Camilo: Y también al cangrejo, que caminaba adelante $y$ lo echaron de la familia.

Coordinadora: Sí, pero hay una cosa que dijo Sara muy importante, los dos estaban solos, el cangrejo tenía familia pero lo echaron.

Sara: Y el perro no tenía familia.

Camilo: Era callejero.

Coordinadora: Era un perro callejero, puede ser, claro.

Sara: Si lo atrapa la perrera lo encierran.

Coordinadora: Ah, pobrecito, claro.

La evocación de la perrera produjo enlaces novedosos con la película de Garfield, conocida por todos ellos. No así por la coordinadora, que quedó ubicada en el lugar del no saber, mientras los niños aportaban detalles y precisiones. Finalmente, la emoción se conectó a la palabra: "Los dos estaban tristes" (Joaquín).

El segundo momento corresponde a lectura de un clásico, "Hansel y Gretel", ocurrida en el décimo cuarto taller. Un aspecto a tomar en cuenta es el escenario de privación y necesidad que padece la familia de los protagonistas, así como su contracara, la abundancia y el exceso representados por la casa de chocolate. La voracidad despertada parece merecedora de un castigo inmediato, a manos de la bruja, un personaje terrorífico que seduce y captura al mismo tiempo, arrojando a los niños a una situación de riesgo vital. Una vez leída, la historia recibió bastantes más rechazos que aceptaciones en el taller. Hablaban de variados tópicos para deshacerse de ella, ignorándola: recordaban todos los cuentos leídos a la fecha y establecían comparaciones desventajosas para el cuento de los hermanos Grimm, intercambiaban puntos de vista e incluso recurrieron a un poema de Rubén Darío que habían aprendido con la maestra de clase. Las dificultades de orden económico formaban parte de la cotidianeidad de las familias a las que pertenecían estos niños. No era sorprendente que surgiera una lluvia de asociaciones referida a sus recientes ingestas, solo en apariencia alejadas del argumento en cuestión. Joaquín, un niño cuya mamá sufría importantes aprietos económicos, fue el primero en hablar del tema, aunque en modo defensivo: "Adivinen qué... Anoche me comí una pizza, toda la pizza de pancho con muzzarella" (Joaquín).

Otros lo secundaron, compitiendo por la cantidad de comida, siempre en calidad de autorreferencias. Entonces se les dijo: “;Ah! Quedaron impactados con el tema del hambre, ¿no? Y ahora vienen a contar todo lo que comieron..." (Coordinadora).

El cuento de Hansel y Gretel convoca asimismo sentimientos de pérdida y abandono, que coincidían con algunas de las historias reales de esos escolares. Tal vez por eso Victoria alegó con gran convicción: “A mí no me gustó nada de ese cuento. ... Porque... porque no me gusta cuando, cuando los niños vi, vie-ron una casa de dulce, y... y apareció la bruja".

No obstante, la actitud refractaria del grupo frente a la historia de Hansel y Gretel fue retomada. Se señaló entonces que ninguno había podido hablar de la parte más triste del cuento y eso movió una traba que permitió que se acercaran un poco más al texto y a sí mismos:

Victoria: ... me gustó cuando, cuando, encontraron al padre.

Braian: Sí, a mí no me gustó la parte cuando se perdieron.

Sara: A mí no me gustó la parte, cuando los dejaban solos.

\section{Dimensiones de análisis y operacionalización de los procesos imaginativo-reflexivos}

Se presenta a continuación un esquema general de las tres dimensiones de análisis con las que se viene trabajando en las diversas ediciones del taller clíniconarrativo y sus respectivas subdimensiones:

Cuadro 1. Dimensiones de análisis

CONSTRUCCIÓN DE ALTERIDAD

[Cuentos y equivalentes concebidos como una otredad a reconocer.

Tratamiento del texto leído en el taller.]

Uso del texto como objeto de conocimiento (Uso / No Uso)

Curiosidad convocada por el texto (Presencial / Ausencia)

Posición subjetiva de cada niño frente

al saber (Saber / No saber)

TRATAMIENTO DEL CONFLICTO FICCIONAL

Mecanismos defensivos diádicos

Mecanismos defensivos triádicos

Mecanismos defensivos diádicos - triádicos

PROCESOS IMAGINATIVO - REFLEXIVOS

Procesos imaginativos

Procesos reflexivos

Como se planteó antes, los procesos imaginativo-reflexivos constituyen el derrotero de este informe. Son una forma particular de uso del objeto, variable de la primera dimensión de análisis. La historia leída por un coordinador del grupo de niños se debe pensar como un eventual objeto de conocimiento que cada uno podrá hacer uso o no, acercándose o distanciándose. 
En primer término habrá de escucharla o desoírla y reconocerla como una otredad o hacer de ella un manojo de proyecciones. En esta última circunstancia, adoptará las características de un objeto subjetivo (Winnicott, 1989/1991); el texto será ignorado o distorsionado en grado extremo. Por el contrario, cuando el cuento ofrecido se puede usar, adquiere las características de un objeto objetivo. Si bien las modalidades de este uso pueden ser variadas, incluso en un mismo sujeto, se han establecido tres alternativas que es necesario recordar y distinguir: uso reproductivo, uso interpretativo y uso creativo (Kachinovsky, 2016).

La relevancia alcanzada por el uso creativo del cuento y de otras producciones simbólicas que circulaban en el taller (canciones, películas invocadas y relatadas, poemas) determinó la necesidad de profundizar en su análisis, tomando en consideración la creación de sentidos subjetivos singulares que hacen a la construcción de textos (Cantú, 2011). Se introdujo una diferenciación operativa entre procesos imaginativos y procesos reflexivos, prestando atención a los diversos enlaces producidos. En virtud de la importancia de las teorizaciones y auto teorizaciones detectadas, se les otorgó un particular tratamiento, más allá de su decidido carácter reflexivo. Se detallan a continuación las variables utilizadas en el análisis de los procesos imaginativos y reflexivos.

\section{Procesos imaginativos}

Los procesos imaginativos fueron operacionalizados como el producto de cualquier enlace entre los siguientes términos:

Tabla 1. Operacionalización de los procesos imaginativos (términos de enlace)

\begin{tabular}{ll}
\hline Término de enlace 1 (variable) & Término de enlace 2 (variable) \\
\hline Decir/hacer de la coordinadora (valor) & Decir/hacer de la coordinadora (valor) \\
Decir/hacer de un par (valor) & Decir/hacer de un par (valor) \\
Decir/hacer de un adulto referente del ámbito & Decir/hacer de un adulto referente del \\
escolar o extraescolar (valor) & ámbito escolar o extraescolar (valor) \\
Decir/hacer propio (valor) & Decir/hacer propio (valor) \\
Cuento leído en el taller (valor) & Cuento leído en el taller (valor) \\
Cuento leído en otros talleres (valor) & Cuento leído en otros talleres (valor) \\
Otras ficciones conocidas (valor) & Otras ficciones conocidas (valor) \\
Otros textos (valor) & Otros textos (valor) \\
Experiencia personal contada (valor) & Experiencia personal contada (valor) \\
Afecto expresado (valor) & Afecto expresado (valor) \\
Otro (valor) & Otro (valor) \\
\hline
\end{tabular}

Algunos ejemplos:

- Enlace producido entre los siguientes términos: cuento leído en el taller-otras ficciones conocidas: Se relaciona un fragmento del cuento aportado por la coordinación con un fragmento de otro cuento u otra versión del mismo cuento escuchados en otro momento, dentro o fuera del taller.

- Enlace producido entre los siguientes términos: cuento leído en el taller-experiencia personal contada: Un fragmento del cuento aportado por la coordinación es asociado al relato de una experiencia personal sucedida dentro o fuera del taller.

- Enlace producido entre los siguientes términos: cuento leído en el taller-afecto expresado:
Un fragmento del cuento aportado por la coordinación es asociado a una expresión verbal o gestual de afecto.

- Enlace producido entre los siguientes términos: experiencia personal contada-afecto expresado: La experiencia personal aportada por un niño es vinculada por el mismo niño que la relata o por otro que la recoge a una expresión verbal de afecto.

\section{Procesos reflexivos}

Los procesos reflexivos fueron operacionalizados como el producto de un enlace entre los siguientes términos: 
Tabla 2. Operacionalización de los procesos reflexivos (términos de enlace)

\begin{tabular}{ll}
\hline Término de enlace 1 (variable) & Término de enlace 2 (variable) \\
\hline Decir/hacer de la coordinadora (valor) & $\begin{array}{l}\text { Cuestionamiento + Interrogante (valor): cuestionamiento } \\
\text { expresado en forma interrogativa } \\
\text { Cuestionamiento + No Interrogante (valor): } \\
\text { cuestionamiento expresado afirmativamente }\end{array}$ \\
$\begin{array}{l}\text { Decir/hacer de un par (valor) } \\
\text { Decir/hacer de un adulto referente del ámbito } \\
\text { escolar o extraescolar (valor) }\end{array}$ & $\begin{array}{l}\text { interrogante sin pretensión de cuestionar } \\
\text { No Cuestionamiento + No Interrogante } \\
\text { (valor): no cuestiona ni pregunta }\end{array}$ \\
Cuento leído en el taller (valor) & Teorización sobre el mundo y los otros (valor) \\
Cuento leído en otros talleres (valor) & Auto teorización o teorización sobre sí mismo (valor) \\
Otras ficciones mencionadas (valor) & \\
Otros textos mencionadas (valor) & \\
Experiencia personal contada (valor) & \\
Afecto expresado (valor) & \\
Otro (valor) & \\
\hline
\end{tabular}

Algunos ejemplos:

- Enlace producido entre los siguientes términos: Decir/hacer de un par - Cuestionamiento + No Interrogante: Un niño discrepa con lo dicho por uno de sus compañeros, ya sea por rivalidad o para anteponer su discurso.

- Enlace producido entre los siguientes términos: Otras ficciones mencionadas - No Cuestionamiento + Interrogante: Una película mencionada en el taller despierta la pregunta de un niño para saber más sobre el argumento o con el objetivo de entenderlo.

- Enlace producido entre los siguientes términos: Cuento leído en el taller - teorización sobre el mundo y los otros: Un fragmento del cuento incita una reflexión sobre cuestiones problemáticas o enigmáticas de la vida.

\section{Descripción de la tarea de análisis}

Se trabajó sobre la transcripción de la producción discursiva registrada en ocasión de diecisiete (17) talleres clínico-narrativos. El taller 1 se analizó en su totalidad (282 turnos de habla) para generar criterios consensuados a seguir durante la tarea de anotación por el grupo de investigadores, por lo que no se lo incluye en este análisis. El taller 12 tampoco se incluye dado que no se trabajó con cuentos en esa oportunidad. Para los otros 15 talleres, se anotaron únicamente los 50 turnos de habla infantil posteriores a la lectura del cuento. Esta decisión se fundamenta en un análisis cualitativo previo a partir del cual el grupo de investigadores entendió que, en general, la participación infantil era mayor inmediatamente después de la lectura de los cuentos.

En un proyecto anterior, se sometió la transcripción a un análisis cualitativo, organizado en torno a las tres dimensiones psíquicas antes detalladas y a sus respectivas subdimensiones, en virtud de su participación en los procesos de pensamiento. En este proyecto dichas dimensiones fueron retomadas, complejizando el modelo. Para ejecutar su adjudicación a cada taller se generó un esquema de anotación elaborado mediante una negociación de sentidos por un grupo interdisciplinario de investigadores. Se practicaron sucesivos ajustes al manual de anotaciones, por medio de un diálogo constante entre los conceptos jerarquizados y el material empírico disponible (la producción discursiva transcripta). De este modo se ajustaron algunos criterios preestablecidos y se construyeron otros nuevos, generando ejemplos prototípicos que enriquecieron el manual de anotaciones. Este largo proceso permitió, por un lado, el acopio de consensos teórico-clínicos. Por otro lado provocó un tránsito imprevisto: el grupo interdisciplinario de investigadores evolucionó hacia lo que podría concebirse como una "comunidad de práctica", en el sentido propuesto por Étienne Wenger (1998).

\section{Herramientas utilizadas para el análisis}

Para tener un registro informático de los datos y facilitar su procesamiento, se emplearon herramientas de código abierto que allanan la tarea. Se utilizó un código programado en Python 2.7 y la biblioteca Natural Language Toolkit (Team NLTK, 2020) para convertir las transcripciones de los talleres en archivos XML que sirvieran de base para una anotación independiente del 
corpus de trabajo. Los archivos XML fueron anotados utilizando el programa MMAX2 (Müller, 2003; Müller, \& Strube, 2006). Se empleó también la suite de herramientas de análisis lingüístico Freeling (Universtat Politecnica de Catalunya) para realizar un análisis morfosintáctico automático.

\section{Resultados}

En este informe se presentan algunos de los resultados obtenidos, circunscribiendo esta entrega a los procesos imaginativo-reflexivos y a la participación de los niños en los talleres clínico-narrativos.

\section{Participación infantil en los talleres clínico-narrativos}

Como puede verse en las gráficas siguientes, la producción discursiva grupal de los niños - medida en turnos de habla- aumentó a medida que se desarrollaron los talleres. Además, si comparamos la producción discursiva de los niños con la de los adultos (coordinadora y asistentes), puede observarse que, a lo largo de los talleres, los niños participaron cada vez más. Visto de otro modo, con el correr del tiempo, los niños necesitaron menor intervención profesional para alcanzar los niveles de participación conseguidos.

Si asumimos que a mayor exposición a los talleres clínico-narrativos debería corresponder una mayor producción infantil (y que este aumento debería sostenerse en el tiempo), entonces, como puede verse en la gráfica anterior, el aumento es importante.

Se desprende de la gráfica anterior que, en promedio, los niños participaron casi un 35\% más que los referentes coordinadores (línea "Media") y que la producción de los primeros pasó de ser prácticamente igual a la de los referentes (cociente cercano a 1) a ser casi un 50\% mayor (cociente cercano a 1,5 ).

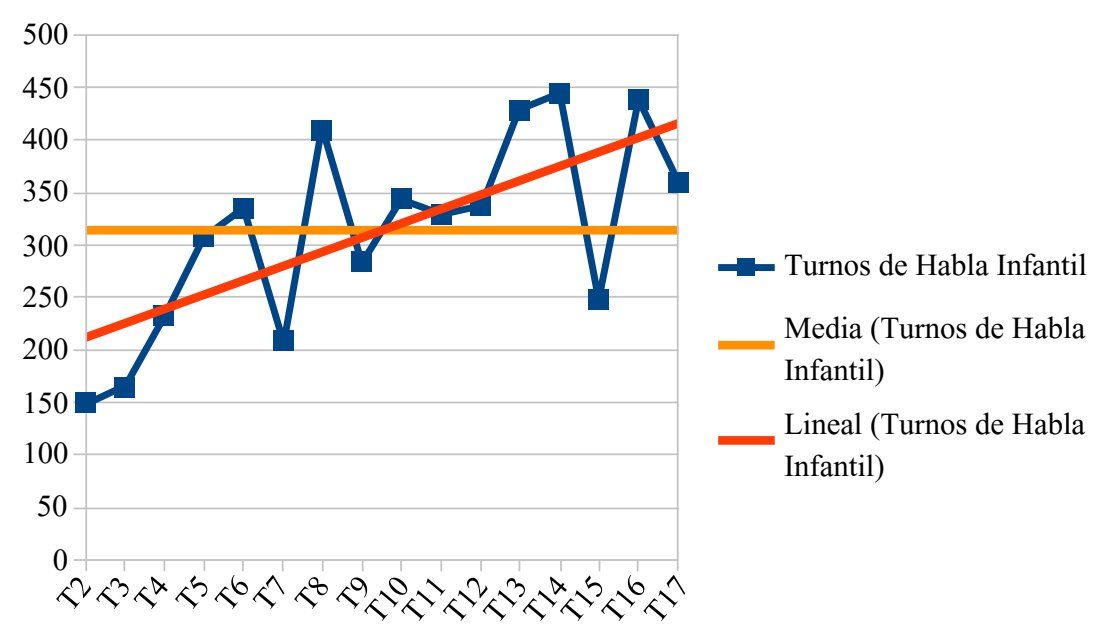

Gráfico 1. Producción infantil según exposición a los talleres

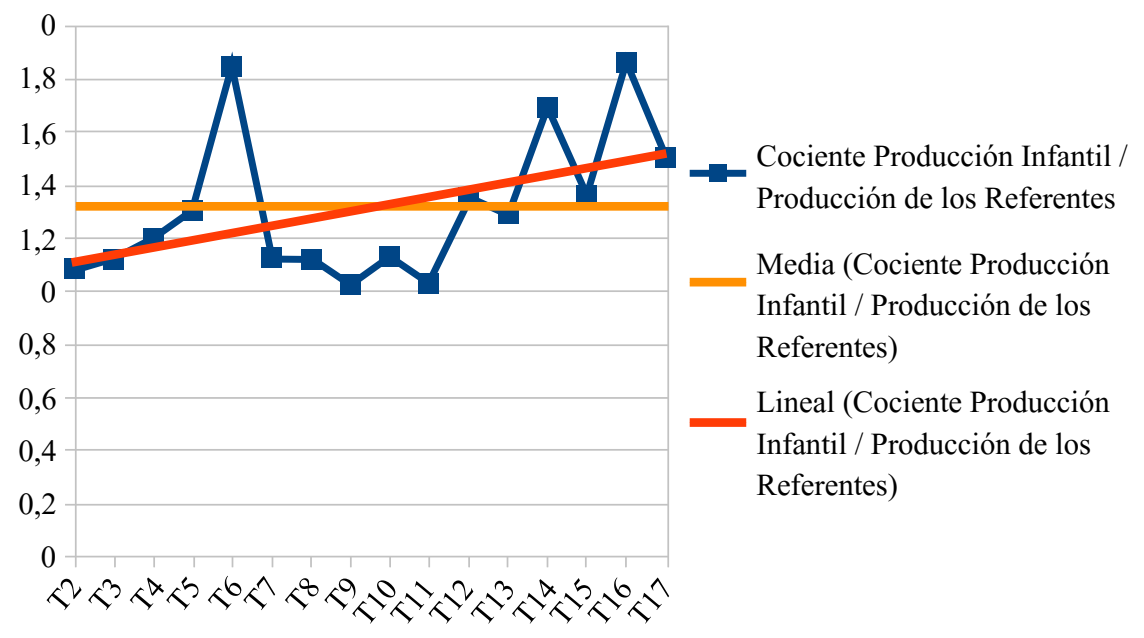

Gráfico 2. Comparación entre participación infantil y participación adulta 


\section{Producción imaginativo-reflexiva en los talleres clínico-narrativos}

Las gráficas siguientes especifican la producción imaginativa y reflexiva de cada niño. En ellas se advierte cierta inclinación a la baja que será justificada en el próximo apartado.

Más allá de lo cuantitativo, los encuentros semanales con la maestra van modificando su mirada y actitudes hacia cada niño. Promueve de año a cinco de los seis niños del taller, impensable a comienzos del año lectivo.

Victoria

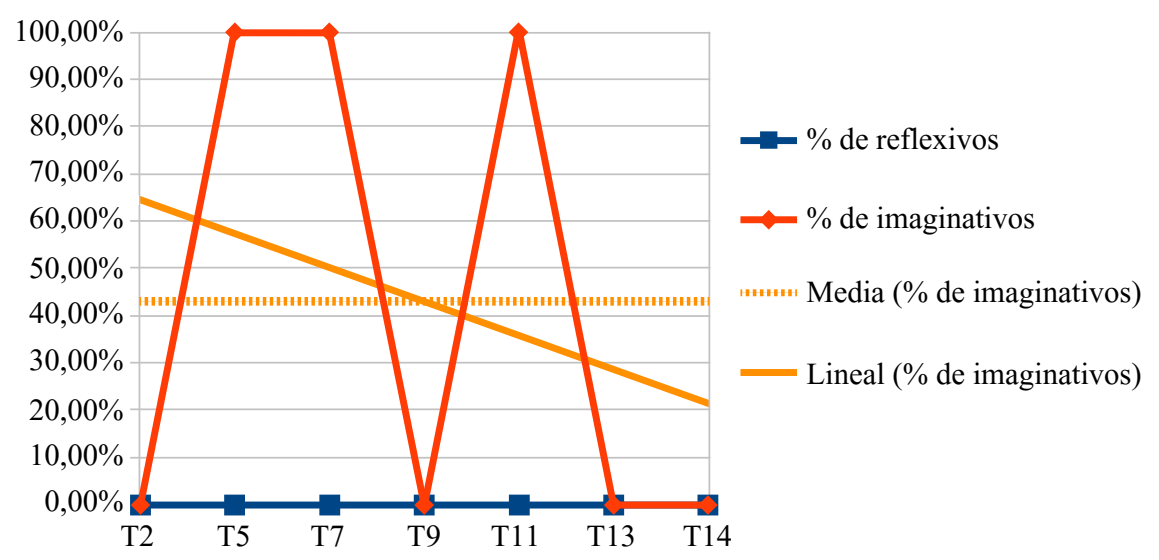

Gráfico 3. Producción imaginativo-reflexiva de Victoria

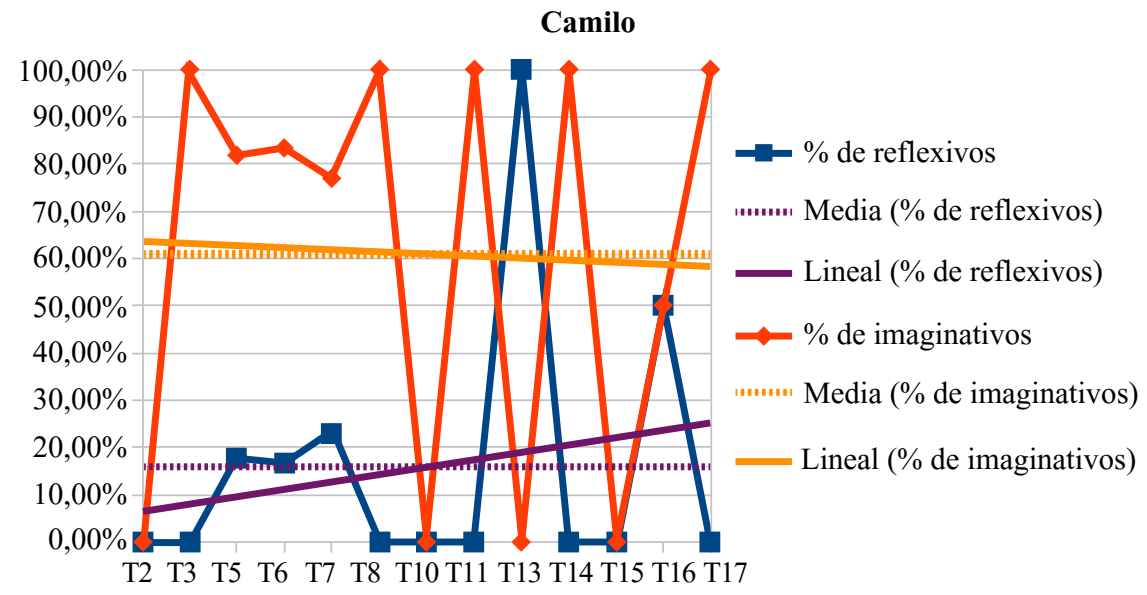

Gráfico 4. Producción imaginativo-reflexiva de Camilo

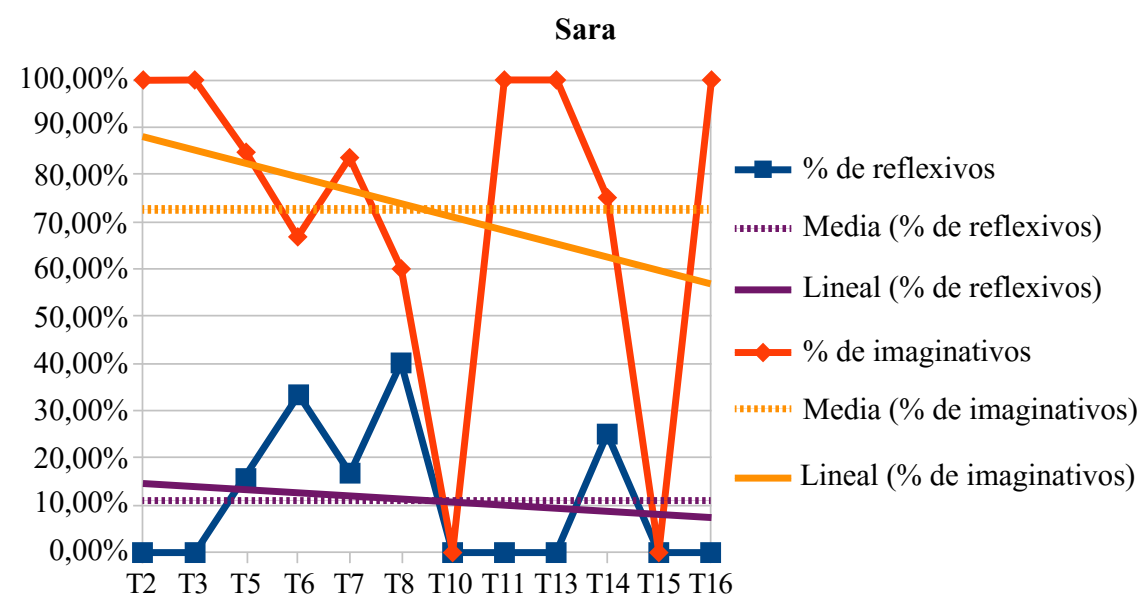

Gráfico 5. Producción imaginativo-reflexiva de Sara 


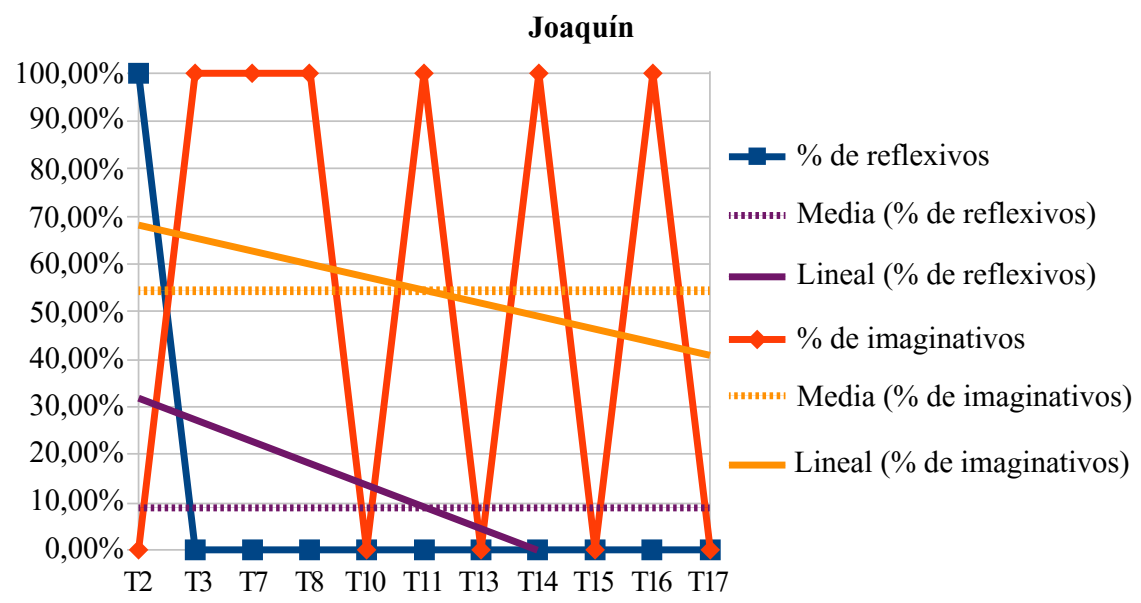

Gráfico 6. Producción imaginativo-reflexiva de Joaquín

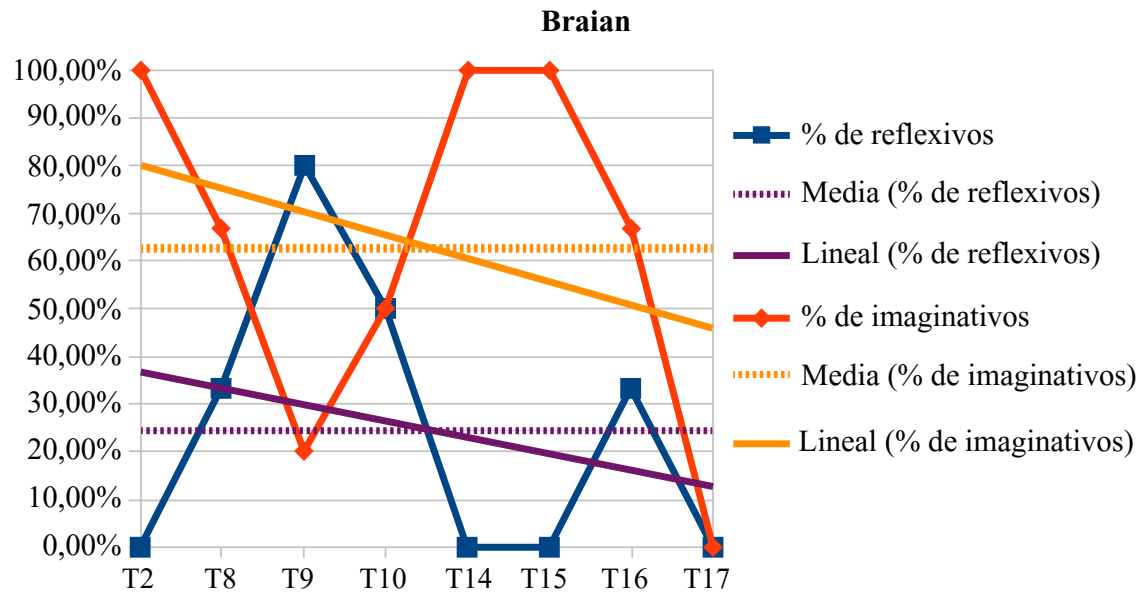

Gráfico 7. Producción imaginativo-reflexiva de Braian

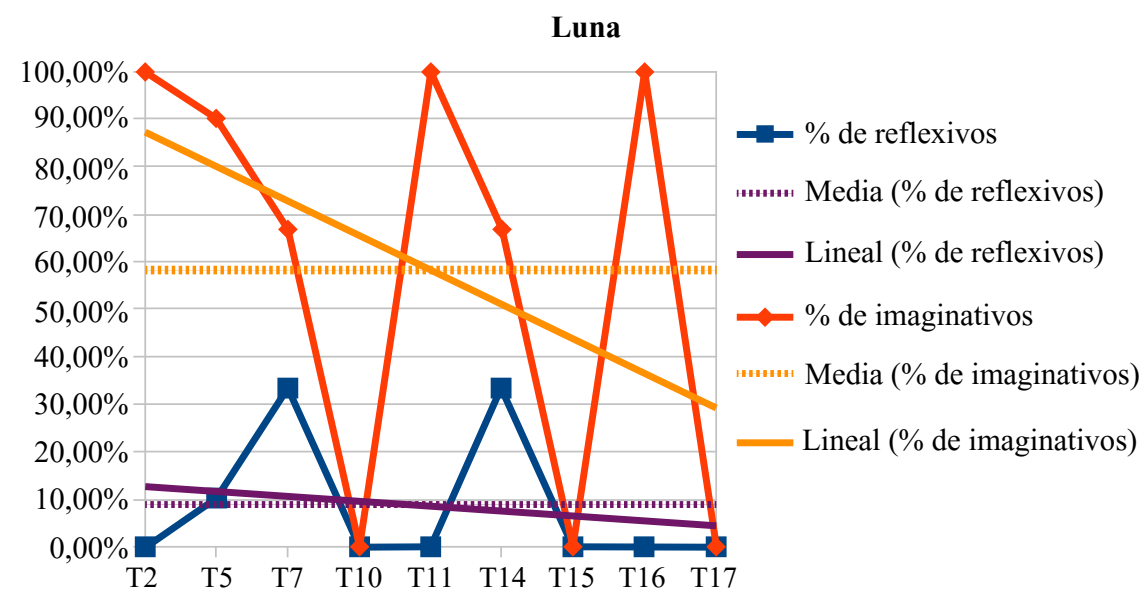

Gráfico 8. Producción imaginativo-reflexiva de Luna

\section{Discusión}

Elasunto pordilucidar en esteapartadoes la tendencia decreciente de la producción imaginativo-reflexiva: ¿por qué los resultados contradicen las expectativas?
Si bien ello podría invalidar las bondades del dispositivo ideado y, en particular, la condición de objeto intermediario del cuento infantil, tal conclusión resulta apresurada y se contradice con otras constataciones. La producción a nivel global aumenta, al tiempo que decaen las 
participaciones de las coordinadoras. Este crecimiento no responde exclusivamente a parámetros cuantitativos. En términos cualitativos se aprecian variaciones significativas: las intervenciones de los niños, antes desordenadas e indiferentes al punto de vista de sus interlocutores (fenómenos pseudocomunicativos), ganan progresivamente estatuto de intercambios conversacionales (fenómenos comunicativos).

Los motivos determinantes del crecimiento negativo de contenidos imaginativos y reflexivos parecen obedecer a muy diversas causas. Entre ellas, cabe destacar:

1. En primer año los niños construyen su identidad escolar, que consiste en la asunción de un conjunto de saberes, prácticas y normas transmitidas por la institución educativa a la que asisten. Hay quienes plantean que este modelado intencional no solo apunta a la disciplina física o a la transmisión de conocimientos sino a suscitar significados unívocos respecto a las prácticas prescriptas por la escuela y al tallado de la subjetividad (Steiner, 2007). Esta adaptación normativa podría incidir asimismo sobre la creatividad domesticándola, es decir, obstaculizando todo acto o pensamiento que contraríe o pervierta lo instituido (en este caso, la versión de cualquier autor).

2. Los niños que tuvieron mayor producción imaginativo-reflexiva desde un punto de vista cuantitativo y cualitativo -Braian, Sara y Joaquín- son aquellos que en forma paulatina pudieron ajustar su comportamiento verbal al encuadre de trabajo instaurado en el taller, en el sentido de respetar la voz de sus compañeros y escucharlos. Dicho logro remite a una creciente regulación emocional, aplazando la urgencia de imponer la palabra y ser escuchado. Este equivalente a las normas de convivencia escolar incluidas en el punto anterior, atribuible al proceso clínico en cuestión, también podría vincularse con los procesos de estructuración psíquica y neogénesis. El consecuente afianzamiento de la represión primaria produciría una restricción de los procesos creativos como derivado secundario, provisorio o permanente.

3. El cuestionamiento oportuno y eventualmente argumentado de todo instituido (texto oficial o escrito leído), así como la capacidad teorizadora, requieren cierta fortaleza yoica habilitante de un descentramiento de la persona propia. Esto permite reconocer el punto de vista de otros - el del autor o el de sus pares en este caso-, asumiéndolo o desechándolo. Precisamente, aquellos niños en los que se observó una menor fortaleza yoica es donde se detecta menor producción imaginativo-reflexiva.

4. Algunas propuestas narrativas de los últimos talleres, como la expresión plástica y la interpretación de canciones en los talleres 12 y 15 respectivamente, tal vez no sean las más aptas para encender controversias o plantear interrogantes en el seno del grupo. Las constantes embestidas e inevitables rupturas del encuadre, y la necesidad de re encuadrar el trabajo del grupo, forjaron la necesidad de promover dinámicas menos exigentes o comprometidas, con el objetivo de aflojar las tensiones grupales y fortalecer la tarea colectiva. Algo similar ocurriría en el último taller, por su carácter de despedida;

5. La coordinadora del taller no disponía en aquel momento de los resultados que acá se presentan, de modo de haber tenido un rol más activo al respecto: proponer más actividades narrativas con el fin de invocar contenidos imaginativos e introducir preguntas, comentarios o señalamientos deliberadamente orientados a la búsqueda del pensamiento reflexivo.

\section{Conclusiones}

La producción discursiva infantil -estimada en turnos de habla- aumentó a medida que se fueron desarrollando los talleres. La comparación de esta producción con la del equipo coordinador fue también favorable; con el correr del tiempo los niños necesitaron una menor intervención de los referentes adultos para alcanzar los niveles mencionados. Cualitativamente, sus aportaciones simbólicas evidenciaron cambios de la intersubjetividad, en tanto los monólogos colectivos de los primeros encuentros fueron reemplazados por fenómenos lingüísticos cooperativos, adquiriendo en ocasiones un carácter especulativo de cuño teorizador.

Se confirma así que a mayor exposición a los talleres clínico-narrativos corresponde una mayor participación infantil sostenida en el tiempo, siendo este incremento importante, amén de avalar la idoneidad del dispositivo creado y la cualidad de objeto intermediario del cuento infantil.

Sin embargo, los números indican una tendencia decreciente de la producción imaginativo-reflexiva, justificada en el apartado anterior por la incidencia de las rutinas y la disciplina escolar, como efecto provisorio o permanente de la intensificación de los procesos de neogénesis (estructuración psíquica) promovidos por el enfoque clínico del taller, por la necesidad de impulsar dinámicas menos exigentes para relajar las tensiones grupales y fortalecer la tarea colectiva, debido a una menguada introducción de preguntas y señalamientos orientados a recabar esta clase de contenidos por parte de la coordinadora.

Por otra parte, la presencia de contenidos reflexivos fue francamente menor que la de contenidos imaginativos. Es muy probable que la producción de interrogantes, cuestionamientos y teorizaciones requiera mayor iniciativa de quienes coordinan el taller. Los cuentos leídos gozan de la condición de un instituido al que sería preciso confrontar. Tienen un carácter de autoridad, porque se sienten como representantes o una prolongación de quienes los escriben o de quienes los seleccionan y relatan.

Tomando en cuenta la producción narrativa registrada en los diferentes talleres, resulta difícil afirmar qué tipo de cuentos, por sí mismos, la concitan más que otros. A diferencia de lo que podría creerse, el dramatismo del argumento no fue obstáculo para 
concitar el interés de los niños ni motivo de rechazo generalizado. Muy por el contrario, los guiones que recuperaban situaciones de infortunio o tragedia devinieron contingencias fecundas en las que se produjeron transformaciones discursivas significativas.

Los debates narrativos que acompañaron la lectura de los cuentos presentaron mayor potencial para complejizar la producción simbólica que el ejercicio de recuperación de personajes y recreación de tramas. En sentido amplio puede decirse que los debates narrativos y otras prácticas no apegadas al libreto oficial constituyeron estrategias particulares de remodelación textual. Propiciaron la apertura del espacio ficcional y comprometieron una mayor implicación del oyente, lo cual invitaba a cada niño a usar las significaciones instituidas (el escrito leído) como punto de partida para reelaborar la trama narrativa y postular nuevas vías de tramitación de las dificultades en juego. Los conflictos ficcionales operaron como activadores de conflictos psíquicos, habilitando un trabajo de perlaboración (Freud, 1914/1976, 1926/1976) o superación de la repetición relacionada con la génesis de nuevos sentidos.

Finalmente, los cambios subjetivos operados en la maestra indican que es posible alterar los efectos de las teorías sociológicas de los 90 implícitas en el cuerpo docente.

\section{Produção imaginativa-reflexiva infantil em oficinas clínico-narrativas}

Resumo: $O$ trabalho centra-se na variável subjetiva do aprendizado: procura identificar as transformações psíquicas de seis crianças em seu trânsito por um dispositivo clínico mediado por contos (oficina narrativa). $O$ trabalho de campo foi realizado em uma escola pública, com crianças de 6 e 7 anos com prognóstico pedagógico desfavorável. Uma ferramenta informática organiza a análise qualitativa e quantitativa da produção discursiva infantil. Os resultados indicam um incremento dessa produção conforme as oficinas foram se desenvolvendo e uma menor exigência de participação da equipe de coordenação. Contudo, observa-se uma tendência decrescente da produção imaginativa-reflexiva que motiva o presente artigo a focalizar nessa dimensão e a dar conta das suas possíveis causas.

Palavras-chave: clínica psicanalítica, crianças, oficina narrativa, processos imaginativo-reflexivos.

\section{Children's imaginative-reflective production in clinical-narrative workshops}

Abstract: The work focuses on the subjective variable of learning: it aims at identifying psychic transformations in six children within a story-mediated clinical setting (narrative workshop). Field work was conducted in a public school, with children aged six and seven characterized by an unfavorable pedagogical prognosis. The qualitative and quantitative analysis of children's discursive production was analyzed using a software tool, with results showing an increase in discursive production throughout the narrative workshops and a decreasing need for the coordinating team's participation. Nevertheless, a decreasing trend in imaginative-reflective production was observed, driving the team to focus the analysis on that dimension and to attempt at explaining its possible causes.

Keywords: psychoanalytic clinic, children, narrative workshop, imaginative-reflective processes.

\section{Production imaginative-réfléchissante pour les enfants dans les ateliers cliniques-narratifs}

Résumé: Le travail se concentre sur la variable subjective de l'apprentissage : il cherche à identifier les transformations psychiques de six enfants en transit à travers un dispositif clinique à médiation narrative (atelier narratif). Le travail de terrain a été réalisé dans une école publique, avec des enfants de 6 et 7 ans, avec un pronostic pédagogique défavorable. Un outil informatique organise l'analyse qualitative et quantitative de la production discursive des enfants. Les résultats montrent une augmentation de cette production au fur et à mesure du développement des ateliers et une moindre exigence de participation de l'équipe de coordination. Cependant, il y a une tendance à la baisse dans la production imaginative-réfléchissante qui nous motive à concentrer ce texte sur cette dimension et à rendre compte de ses causes possibles.

Mots-clés: clinique psychanalytique, enfants, atelier narratif, processus imaginatifs-réflexifs. 


\section{Referencias}

Administración Nacional de Educación Pública, \& Unesco. (2009). Plan Nacional de Educación (2010 - 2030). Montevideo: Anep; Unesco. Recuperado de https:// bit.ly/2GGrrhS

Álvarez, P. (2010). Los trabajos psíquicos del discurso. Buenos Aires: Teseo.

Bernstein, B. (1989). Clases, códigos y control I: Estudios teóricos para una sociología del lenguaje. Madrid: Akal.

Bruner, J. (1991). Actos de significado: Más allá de la revolución cognitiva. (J. C. Gómez Crespo \& J. L. Linaza, trad.). Madrid: Alianza. (Trabalho original publicado em 1990).

Bruner, J. (2003). La fábrica de historias: Derecho, literatura, vida (L. Padilla, trad.). Buenos Aires: Fondo de Cultura Económica. (Trabalho original publicado em 2002).

Cantú, G. (2011). Lectura y subjetividad en el diagnóstico psicopedagógico. Buenos Aires: Noveduc.

Castoriadis, C. (1993). Lógica, imaginación, reflexión. In R. Dorey. El inconsciente y la ciencia (J. L. Etcheverry, trad., pp. 21-50). Buenos Aires: Amorrortu. (Trabalho original publicado em 1991).

Freud, S. (1976). Inhibición, síntoma y angustia. Obras completas (J. L. Etcheverry, trad., vol. 20, pp. 71-164). Buenos Aires: Amorrortu. (Trabalho original publicado em 1926).

Freud, S. (1976). Repetir, recordar y reelaborar. In S. Freud. Obras completas (J. L. Etcheverry, trad., vol. 12, pp. 145-157). Buenos Aires: Amorrortu. (Trabalho original publicado em 1914).

Green, A. (1996). La metapsicología revisitada (I. Agoff, trad.). Buenos Aires: Eudeba. (Trabalho original publicado em 1995).

Green, A. (1999). Sobre la discriminación e indiscriminación afecto-representación. Revista de Psicoanálisis, 56(1), 11-71.

Green, A. (2005). Ideas directrices para un psicoanálisis contemporáneo: Desconocimiento y reconocimiento del inconsciente (L. Lambert, trad.). Buenos Aires: Amorrortu. (Trabalho original publicado em 2003)

Gutfreind, C. (2003). O terapeuta e o lobo: A utilização do conto na psicoterapia da criança. San Pablo: Casa do Psicólogo.

Gutfreind, C. (2007). El potencial terapéutico de los cuentos infantiles: Aplicaciones en psiquiatría infantil. Revista de APPIA, (16), 130-138. Recuperado de https:// bit.ly/369LUUN

Hernández Sampieri, R., Fernández Collado, C., \& Baptista Lucio, P. (2010). Metodología de la investigación (5a ed.). Nova York: Mc Graw Hill.

Kachinovsky, A. (2012). Enigmas del saber: historias de aprendices. Montevideo: Universidad de la República.

Kachinovsky, A. (2014). El cuento infantil como objeto intermediario en la complejización del psiquismo. (Tese de Doutorado). Universidad de Buenos Aires.
Kachinovsky, A. (2016). El cuento infantil como objeto intermediario para el psiquismo. Investigaciones en Psicología, 21(1), 35-44. Recuperado de https:// bit.ly/38kS1si

Martinis, P. (2013). Educación, pobreza y seguridad en el Uruguay de la década de los noventa. Montevideo: Universidad de la República.

Müller, C. (2003). MMAX2 Annotation Tool [Software]. Recuperado de https://bit.ly/36b1qQv

Müller, C., \& Strube, M. (2006). Multi-level annotation of linguistic data with MMAX2. In S. Braun, K. Kohn, \& J. Mukherjee (Eds.), Corpus technology and language pedagogy: New resources, new tools, new methods (pp. 197-214). Berna: Peter Lang.

Team NLTK. (2020). Natural Language Toolkit (versão 3.5) [Software]. Recuperado de https://bit.ly/38ggDlS

Schlemenson, S. (2009). La clínica en el tratamiento psicopedagógico. Barcelona: Paidós.

Patto, M. H. S. (1988). O fracasso escolar como objeto de estudo: anotações sobre as características de um discurso. Cadernos de Pesquisa, (65), 72-77. Recuperado de https://bit.ly/2V2tvUZ

Steiner, E. (2007). Encargos y demandas a la institución escolar, vistos a través de sus mitos, prácticas ritualizadas y objetos simbólicos. Informe final. Montevideo: CSIC; Udelar.

Wald, A. (2015). El concepto de imaginación en psicoanálisis. Aportes teóricos a partir de problemáticas clínicas. Artigo apresentado no VII Congreso Internacional de Investigación y Práctica Profesional en Psicología, Buenos Aires. Recuperado de https://bit.ly/36b5hgr

Warnock, M. (1978). Imagination. Berkeley: University of California Press.

Warnock, M. (1994). Imagination and time. Hoboken: Blackwell.

Wenger, E. (1998). Communities of practice. Learning, meaning and identity. Nova York: Cambridge University Press.

Winnicott, D. W. (1991). Exploraciones psicoanalíticas II (L. Wolfson, trad.). Barcelona: Paidós. (Trabalho original publicado em 1989).

Xirau, R. (2018). Mary Warnock, imagination. Crítica. Revista Hispanoamericana de Filosofía, 12(35), 103-113. doi: 10.22201/iifs. 18704905 e. 1980.340 